\title{
Performance of a Two-Headed Disk System when Serving Database Queries Under the Scan Policy
}

\section{Y. MANOLOPOULOS}

\author{
Aristotelian University of Thessaloniki
}

and

\author{
J. G. KOLLIAS \\ National Technical University of Athens
}

\begin{abstract}
Disk drives with movable two-headed arms are now commercially available. The two heads are separated by a fixed number of cylinders. A major problem for optimizing disk head movement, when answering database requests, is the specification of the optimum number of cylinders separating the two heads. An earlier analytical study assumed a FCFS model and concluded that the optimum separation distance should be equal to 0.44657 of the number of cylinders $N$ of the disk. This paper considers that the SCAN scheduling policy is used in file access, and it applies combinatorial analysis to derive exact formulas for the expected head movement. Furthermore, it is proven that the optimum separation distance is $N / 2-1(\Gamma N / 2-11$ and $(N / 2-1 \mathrm{~J})$ if $N$ is even (odd). In addition, a comparison with a single-headed disk system operating under the same scheduling policy shows that if the two heads are optimally spaced, then the mean seek distance is less than one-half of the value obtained with one head. In fact that the SCAN policy is used for many database applications (for example, batching and secondary key retrieval) demonstrates the potential of two-headed disk systems for improving the performance of database systems.
\end{abstract}

Categories and Subject Descriptors: D.4.2 [Operating Systems]: Storage Management-secondary storage devices; H.2.4 [Database Management]: Systems-query processing

General Terms: Algorithms, Performance

Additional Key Words and Phrases: Batching, disk design, performance evaluation, secondary key retrieval, seek distance, seek time, two-headed disk

\section{INTRODUCTION}

A number of scheduling policies have been evaluated in the past for conventional disk systems with one head per movable arm [25, pp. 314-324]. Disk systems with two heads per moving arm and a fixed number of cylinders between the two heads are now commercially available. These systems aim to minimize the

This work is part of KRINO, a project aimed at improving software technology in Greece.

Authors' current addresses: Y. Manolopoulos, Aristotelian Univeristy of Thessaloniki, Department of Electrical Engineering, Division of Electronics and Computer Engineering, 54006 Thessaloniki, Greece; J. G. Kollias, National Technical University of Athens, Department of Electrical Engineering, Division of Computer Science, 15773 Zografou, Athens, Greece.

Permission to copy without fee all or part of this material is granted provided that the copies are not made or distributed for direct commercial advantage, the ACM copyright notice and the title of the publication and its date appear, and notice is given that copying is by permission of the Association for Computing Machinery. To copy otherwise, or to republish, requires a fee and/or specific permission.

(C) 1989 ACM 0362-5915/89/0900-0425 $\$ 01.50$ 
expensive arm movement and consequently reduce the seek time. An interesting problem related to the performance of two-headed disks is the determination of the optimum number of cylinders separating the two heads. To our knowledge, the only two studies addressing this problem are the ones in [3] and [20] which emphasize conditions found within operating systems. A common characteristic of these studies is that they can be readily applied to the following, among others, disk devices: Burroughs FD210, DEC RA81 and SA82, IBM 3380, and Sperry Univac 8450 and 8470.

The work in [20] examined two versions of a two-headed disk system with $N$ cylinders using a simulation model. The model was driven by a uniform random request sequence and a Shortest Seek Time First (SSTF) scheduling policy. The first version assumed that both heads were constrained to remain within the disk surface, that is, neither head can go beyond cylinder 1 or $N$. In this case the optimum head separation distance was found to be approximately $N / 2$. The second version assumed that either of the two heads may move out of the disk surface, that is, the maximum head traversal is $N-1$ cylinders. Under this case the optimum head separation distance was found to be a linearly reducing function of the queue length. In addition, it was argued that if the SCAN policy is used, then the optimum head separation is $N / 2$. The analytical work in [3] assumed that the requests are distributed over the continuous interval $[0,1]$ and are served on a first come, first served (FCFS) basis under the nearestserver rule. The conclusion reached was that the optimum head separation is $0.44657 * N$, and in this case the expected head movement between successive requests is $0.16059 * N$. The fact that the same expected head movement of single-headed disk systems is approximated by $N / 3$ [24] justifies the conclusion reached in $[3$, p. 826] that "A system with two heads performs more than twice as well as a system with a single head." Two more citations exhaust the literature on disk systems with two heads at a fixed distance. In [4] the problem of optimal directory placement is examined with respect to the minimization of the expected movement of the moving arm. Similarly, the problem of optimal placement of file records has been solved in [17] by taking into account the record access probabilities. This optimal placement looks like two successive organ-pipe arrangements and is called a camel arrangement. The number of optimal camel arrangements is also derived in [18]. In [13] a disk system with two independent arms is considered, and it is shown that the seek time is minimized when one arm serves the request and the other jockeys for optimal anticipatory position.

This paper studies the performance of a two-headed disk system which operates under the SCAN scheduling policy. This policy moves the heads in a consistent direction when serving queued database requests. As shown in [2], [15], [18], and [24] the SCAN policy is an efficient method for satisfying either a batch of queries based on primary key values [22] or one query based on secondary key values. The reason is that in both cases the query may be satisfied by monotonically accessing the cylinders where the primary file records reside. The overheads involved for performing the strategy are normally insignificant. For example, secondary indexes already have their references to the primary files ordered in an ascending sequence of cylinder numbers.

One may further argue that a number of Data Manipulation Language (DML) statements of popular Database Management Systems (DBMS) may be 
efficiently implemented using the SCAN policy. Consider, for example, the disk head movements involved when the statement FIND NEXT, which locates the members of a given owner, is executed on a network model DBMS [10]. If the member records are ordered and placed on the disk according to their primary key values, then the execution of the statement may be accelerated when the SCAN policy is used. In fact, network databases offer the Data Definition Language (DDL) statement

KEY IS ASCENDING 〈primary-key〉 IN (set-name)

which allows the implementation of the proposed placement [10]. Similar arguments apply when processing hierarchical databases, for example, the IMS DBMS [10]. Finally, we note that the SCAN policy remains a useful accessing strategy even when record insertions and deletions have destroyed the monotonocity of the cylinder accesses. Database reorganization restores the initial performance $[12,21,23]$.

This paper consists of five further sections. Section 2 presents some definitions and assumptions and lists some known results from previous studies on oneheaded systems. Section 3 introduces and explains the way SCAN operates in a two-headed disk system. Section 4 uses combinatorial analysis to estimate the exact expected head movement to answer a batch of some requests as a function of the number of cylinders of the system, the head separation distance, and the size of the batch. Section 5 proves that the optimum head separation distance is $N / 2-1(\lceil N / 2-1\rceil$ and $L N / 2-1\rfloor)$ if $N$ is even (odd). Furthermore, comparison with a one-headed disk system operating under the same scheduling policy shows reduction of over 50 percent in seek distance covered. In the last section some conclusions are stated and certain suggestions for further research are made.

\section{ASSUMPTIONS AND DEFINITIONS}

For the rest of the paper we consider a primary file occupying $N$ consecutive cylinders of a two-headed disk system. The system has to locate $R$ file records, where $R$ is the size of the batch or the number of records satisfying a query based on secondary attributes. The following assumptions are made:

(1) To answer the user query $Q$ distinct cylinders must be visited, where $Q \leq R$ and $1 \leq Q \leq N$;

(2) All cylinders are equally probable to be visited. It should be noted that this assumption is the most commonly used, although it may not be satisfied in actual database systems. The reasons are that (a) it makes mathematical analysis easier and produces upper bounds on the expected distance [8] and (b) it is the most reasonable one in the absence of any other information;

(3) The $Q$ cylinders are contained in a list which is sorted on the ascending cylinder number, and the cost of maintaining the list is negligible;

(4) The first (left) head is initially positioned above the first cylinder, and the second (right) head is positioned over the $(D+2)$ th cylinder. The disk head separation distance $D$ is the number of cylinders between the cylinders which have heads above them. Clearly, $0 \leq D \leq N-2$. This assumption implies that 
one and only one disk head may move out of the recording area. In [20] the two cases in which the disk heads may or may not move beyond the recording area are examined separately. In the present report both cases are examined together.

The scheduling policy to be used is the SCAN algorithm. According to SCAN (as applied to database searching in one-headed systems), the disk heads move from the first to the last cylinder alternatively. Each time, either a batch of queries (based on primary key values) or one query (based on secondary key values) is satisfied [15]. If more than one such query is involved, then the system satisfies them either on a FCFS basis [2] or according to an optimization algorithm $[2,16,18,26]$. In other words, the algorithm rules out techniques that would interleave searching for more than one query in one scanning of the disk.

At this point, we relate the most relevant results for single-headed disk systems. In [15] the seek time for batched searching of random or index sequential files is given by the equation

$$
T=Q S \min +D T(S \max -S \min ) /(N-1)
$$

where $S \min (S \max )$ is the seek time when the disk heads are moving a distance of $1(N-1)$ cylinder(s) and $D T$ is the expected distance traveled by the disk heads. A number of papers have been published on the estimation of $Q$; among others we note [5-7], [19], [27], and [28]. In [18] the SCAN policy was also considered, and it was proven that the expected head movement to visit all $Q$ requests is

$$
D T=\frac{N Q-1}{Q+1} \text {. }
$$

This relation shows that a batch of $Q$ visited cylinders causes approximately $Q /(Q+1)$ portion of the file to be searched. We note also that similar formulas have been obtained in [16]. Results concerning single headed disk systems are discussed again in Section 5.

We call the number of cylinders between any two specific cylinders a subinterval.

LEMма [9]. The probability distribution of the length of the subintervals between $(a)$ any two successively hit cylinders, or $(b)$ the beginning of the file and the first hit cylinder, or (c) the last hit cylinder and the end of the file is

$$
P(N, Q, K)=\frac{\left(\begin{array}{c}
N-K-1 \\
Q-1
\end{array}\right)}{\left(\begin{array}{c}
N \\
Q
\end{array}\right)}
$$

where $N$ is the total number of cylinders, $K$ is the size of the subinterval, and $Q$ is the number of visited cylinders.

Obviously, $P(N, Q, K)$ takes positive real values for $N \geq Q$ and $0 \leq K \leq N-Q$. In all other cases this distribution equals zero. In fact only the third type of subinterval is used in the mathematical analysis to follow, and it is called the final subinterval. 
Before proceeding further we note that in the next section we assume that the following relations are known [11, 14]:

$$
\begin{aligned}
\sum_{K=1}^{N-M}\left(\begin{array}{c}
N-K \\
M
\end{array}\right) & =\left(\begin{array}{c}
N \\
M+1
\end{array}\right) \\
\sum_{K=1}^{N-M} K\left(\begin{array}{c}
N-K-1 \\
M-1
\end{array}\right) & =\left(\begin{array}{c}
N \\
M+1
\end{array}\right) \\
\sum_{K=0}^{N}\left(\begin{array}{l}
R \\
K
\end{array}\right)\left(\begin{array}{c}
P \\
N-K
\end{array}\right) & =\left(\begin{array}{c}
R+P \\
N
\end{array}\right)
\end{aligned}
$$

\section{SCAN APPLIED IN TWO-HEADED DISK SYSTEMS}

In this section, we give an example that explains the way SCAN operates in twoheaded systems. This example also eases the comprehension of the mathematical analysis of the following section.

Example. Suppose that $N=9, Q=2$, and $D=3$. Figure 1 shows the $\left(\begin{array}{l}9 \\ 2\end{array}\right)=36$ combinations of requesting 2 cylinders out of 9 which the file occupies. The 36 cases are equally likely to occur. The crosses in columns indicate that the specific cylinders are visited. At the right side of the figure the necessary head movement is depicted. Initially the left (right) head is on cylinder 1 (5). This explains why the head movement is 0 in case 4 and 1 in cases 1 and 27. In fact the left (right) head serves both requests in case 1 (27).

Consider now the variable $K$, an integer nonnegative number which represents the number of consecutive cylinders at the end of the disk pack that are not visited (the final subinterval). Note that in our example $K$ may vary from 0 to 7 . There are three possible cases, namely:

(1) The rightmost hit cylinder address is smaller than $D+2$ (for our example $D+2=5$ ). In this case all the requests fall in the range 1 st to 4 th cylinder and the left (right) head answers all requests (no request). The final subinterval, $K$, may range from 7 to 5 . It may not be greater than 7 because according to the first assumption, the requests hit the first two cylinders.

(2) The rightmost hit cylinder address is an element of $[2 D+2, N]$ (for our example $[8,9]$ ). In this case the subinterval between the last hit cylinder and the cylinder on the top of which the right head was positioned initially is not shorter than $D$. This implies that if the right head has satisfied the rightmost request, then the left head will have passed over the cylinder on the top of which the right head was initially positioned. Therefore, all requests have been answered. The subinterval $K$ lies in the range 1 to 0 .

(3) The rightmost hit cylinder address is an element of $[D+2,2 D+1]$ (for our example $[5,7]$ ). In this case the subinterval between the rightmost hit cylinder and the cylinder on the top of which the right head was positioned initially is shorter than $D$, say $D^{\prime}$. Under this condition, there may be a possibility that a number of requests have not been satisfied. These requests may concern the $D-D^{\prime}$ consecutive cylinders to the left of the original position of the right 


\begin{tabular}{|c|c|c|c|c|c|c|c|c|c|c|c|}
\hline & \multicolumn{9}{|c|}{ Cylinder number } & \multirow{2}{*}{$\begin{array}{c}\text { Final } \\
\text { subinterval }\end{array}$} & \multirow{2}{*}{$\begin{array}{c}\text { Head } \\
\text { movement }\end{array}$} \\
\hline & 1 & 2 & 3 & 4 & 5 & 6 & 7 & 8 & 9 & & \\
\hline $\begin{array}{r}1 \\
2 \\
3 \\
4 \\
5 \\
6 \\
7 \\
8 \\
9 \\
10 \\
11 \\
12 \\
13 \\
14 \\
15 \\
16 \\
17 \\
18 \\
19 \\
20 \\
21 \\
22 \\
23 \\
24 \\
25 \\
26 \\
27 \\
28 \\
29 \\
30 \\
31 \\
32 \\
33 \\
34 \\
35 \\
36\end{array}$ & $\begin{array}{l}+ \\
+ \\
+ \\
+ \\
+ \\
+ \\
+ \\
+\end{array}$ & $\begin{array}{l}+ \\
+ \\
+ \\
+ \\
+ \\
+ \\
+\end{array}$ & $\begin{array}{l}+ \\
+ \\
+ \\
+ \\
+ \\
+\end{array}$ & $\begin{array}{l}+ \\
+ \\
+ \\
+ \\
+\end{array}$ & $\begin{array}{l}+ \\
+ \\
+ \\
+\end{array}$ & $\begin{array}{l}+ \\
+ \\
+\end{array}$ & $\begin{array}{l}+ \\
+\end{array}$ & + & + & $\begin{array}{l}7 \\
7 \\
5\end{array}$ & $\begin{array}{l}1 \\
2 \\
3\end{array}$ \\
\hline
\end{tabular}

Fig. 1. The necessary head movement for $N=9, D=3$, and $Q=2$.

head. Therefore, additional disk head movement may be required in order to satisfy some requests by the left head. In this case $K$ lies in the range 4 to 2 .

By means of the variable $K$, which in our example takes all the possible values and only once, it is easily understood that the three cases are mutually exclusive and exhaustive. In the following section all the possibilities for each case in the example are enumerated.

\section{ANALYSIS OF DISK HEAD MOVEMENT}

In this section we concentrate on the analysis of the expected distance traveled, that is, the factor DT in relation (1). In the next section we discuss the impact of the selectivity $Q$ in the total seek time.

\subsection{First Case: $N-Q \geq K \geq N-D-1$}

This case is met when the rightmost hit cylinder lies to the left of the initial position of the right head. For our example, this condition may be interpreted as 
$7 \geq K \geq 5$, and it corresponds to lines $1,2,3,9,10$, and 16 of Figure 1 . The expected head movement to answer these permutations is

$$
\sum_{K=N-D-1}^{N-Q}(N-1-K) P(N, Q, K)
$$

Using $P(N, Q, K)$ as given by (3) we obtain

$$
\frac{1}{\left(\begin{array}{l}
N \\
Q
\end{array}\right)} \sum_{K=N-D-1}^{N-Q}(N-1-K)\left(\begin{array}{c}
N-1-K \\
Q-1
\end{array}\right) .
$$

By substituting (4) and (5) and simplifying, the relation becomes

$$
\frac{(D+1) Q-1}{Q+1} \frac{\left(\begin{array}{c}
D+1 \\
Q
\end{array}\right)}{\left(\begin{array}{l}
N \\
Q
\end{array}\right)}
$$

\subsection{Second Case: $N-2-2 D \geq K \geq 0$}

This case is met whenever the right head travels at least a distance equal to $D$ to answer the rightmost request. For our example, this condition $(1 \geq K \geq 0)$ corresponds to lines $7,8,14,15,20,21,25,26,29,30,32,33,34,35$, and 36 . It can be easily seen that if $N-2 D-2<0$, then the following analysis does not contribute to the final result. The expected head movement is

$$
\begin{aligned}
& \sum_{K=0}^{N-2-2 D}(N-D-2-K) P(N, Q, K) \\
& \quad=\frac{1}{\left(\begin{array}{l}
N \\
Q
\end{array}\right)} \sum_{K=0}^{N-2-2 D}(N-D-2-K)\left(\begin{array}{c}
N-1-K \\
Q-1
\end{array}\right)=\mathrm{A} .
\end{aligned}
$$

Without loss of generality let $Q \leq 2 D+2$. If this condition is not true, then some combinations are equal to zero and do not contribute to the result of this subsection. This proposition will be shown up later by considering that the result contains the combination $\left({ }^{2 D+1}{ }_{Q}^{1}\right)$. Then:

$$
\begin{aligned}
& \mathrm{A}=\frac{1}{\left(\begin{array}{l}
N \\
Q
\end{array}\right)}\left\{(N-D-2)\left[\sum_{K=0}^{N-Q}\left(\begin{array}{c}
N-1-K \\
Q-1
\end{array}\right)-\sum_{K=N-2 D-1}^{N-Q}\left(\begin{array}{c}
N-1-K \\
Q-1
\end{array}\right)\right]\right. \\
& \left.-\left[\sum_{K=1}^{N-Q} K\left(\begin{array}{c}
N-1-K \\
Q-1
\end{array}\right)-\sum_{K=N-2 D-1}^{N-Q} K\left(\begin{array}{c}
N-1-K \\
Q-1
\end{array}\right)\right]\right\} .
\end{aligned}
$$

ACM Transactions on Database Systems, Vol. 14, No. 3, September 1989. 
We now have that

$$
\begin{aligned}
& \sum_{K=N-2 D-1}^{N-Q} K\left(\begin{array}{c}
N-1-K \\
Q-1
\end{array}\right) \\
& \quad=(N-2 D-2) \sum_{K=N-2 D-1}^{N-Q}\left(\begin{array}{c}
N-1-K \\
Q-1
\end{array}\right)+\sum_{K=1}^{2 D+2-Q} K\left(\begin{array}{c}
2 D+1-K \\
Q-1
\end{array}\right) \\
& \quad=(N-2 D-2)\left(\begin{array}{c}
2 D+1 \\
Q
\end{array}\right)+\left(\begin{array}{c}
2 D+2 \\
Q+1
\end{array}\right) .
\end{aligned}
$$

By substituting (3), (4), and (8) the relation becomes

$$
\begin{aligned}
\mathrm{A}= & \frac{1}{\left(\begin{array}{c}
N \\
Q
\end{array}\right)}\left\{(N-D-2)\left[\left(\begin{array}{l}
N \\
Q
\end{array}\right)-\left(\begin{array}{c}
2 D+1 \\
Q
\end{array}\right)\right]\right. \\
& \left.-\left[\left(\begin{array}{c}
N \\
Q+1
\end{array}\right)-(N-2 D-2)\left(\begin{array}{c}
2 D+1 \\
Q
\end{array}\right)-\left(\begin{array}{c}
2 D+2 \\
Q+1
\end{array}\right)\right]\right\} \\
= & \frac{1}{\left(\begin{array}{c}
N \\
Q
\end{array}\right)}\left\{\left(\frac{(N+1) Q}{Q+1}-D-2\right)\left(\begin{array}{l}
N \\
Q
\end{array}\right)+\left(\frac{2 D+2}{Q+1}-D\right)\left(\begin{array}{c}
2 D+1 \\
Q
\end{array}\right)\right\} .
\end{aligned}
$$

If $Q>2 D+2$, then the analysis is straightforward and yields that the expected head movement is $(N+1) Q /(Q+1)-D-2$.

\subsection{Third Case: $N-D-2 \geq K \geq N-2 D-1$}

This case occurs whenever the right head travels a distance shorter than $D$ to answer the rightmost request. For our example, this condition $(4 \geq K \geq 2$ ) corresponds to lines $4,5,6,11,12,13,17,18,19,22,23,24,27,28$, and 31 . This case is divided in two subcases. First, we estimate the expected movement of the arm so that the second head accesses all the cylinders lying to its right. Second, we estimate the expected movement of the arm so that the first head accesses any cylinder not yet visited. The latter subcase corresponds to lines $11,17,18$, 22,23 , and 24 . Note that if $N-2 D-2<0$ but $N-D-2 \geq 0$, then the valid range of $K$ is $N-D-2 \geq K \geq 0$. The analysis for this condition follows later in this subsection.

$$
\begin{aligned}
& \sum_{K=N-2 D-1}^{N-D-2}(N-D-2-K) P(N, Q, K) \\
& \quad=\frac{1}{\left(\begin{array}{l}
N \\
Q
\end{array}\right)} \sum_{K=N-2 D-1}^{N-D-2}(N-D-2-K)\left(\begin{array}{c}
N-1-K \\
Q-1
\end{array}\right)=\mathrm{B} .
\end{aligned}
$$

As in the previous subsection, let $D+2>Q$ without loss of generality. If this condition does not hold, then the analysis is easy to follow and is 
therefore omitted. Then

$$
\begin{aligned}
\mathrm{B}=\frac{1}{\left(\begin{array}{l}
N \\
Q
\end{array}\right)}\left\{(N-D-2)\left[\sum_{K=N-2 D-1}^{N-Q}\left(\begin{array}{c}
N-1-K \\
Q-1
\end{array}\right)-\sum_{K=N-D-1}^{N-Q}\left(\begin{array}{c}
N-1-K \\
Q-1
\end{array}\right)\right]\right. \\
\left.-\left[\sum_{K=N-2 D-1}^{N-Q} K\left(\begin{array}{c}
N-1-K \\
Q-1
\end{array}\right)-\sum_{K=N-D-1}^{N-Q} K\left(\begin{array}{c}
N-1-K \\
Q-1
\end{array}\right)\right]\right\} .
\end{aligned}
$$

We now have that

$$
\begin{aligned}
& \sum_{K=N-D-1}^{N-Q} K\left(\begin{array}{c}
N-1-K \\
Q-1
\end{array}\right) \\
& \quad=(N-D-2) \sum_{K=N-D-1}^{N-Q}\left(\begin{array}{c}
N-1-K \\
Q-1
\end{array}\right)+\sum_{K=1}^{D+2-Q} K\left(\begin{array}{c}
D+1-K \\
Q-1
\end{array}\right) \\
& \quad=(N-D-2)\left(\begin{array}{c}
D+1 \\
Q
\end{array}\right)+\left(\begin{array}{c}
D+2 \\
Q+1
\end{array}\right) .
\end{aligned}
$$

By combining (4), (8), and (11) it is derived that

$$
\begin{aligned}
\mathrm{B}= & \frac{1}{\left(\begin{array}{c}
N \\
Q
\end{array}\right)}\left\{(N-D-2)\left[\left(\begin{array}{c}
2 D+1 \\
Q
\end{array}\right)-\left(\begin{array}{c}
D+1 \\
Q
\end{array}\right)\right]\right. \\
& -\left[(N-2 D-2)\left(\begin{array}{c}
2 D+1 \\
Q
\end{array}\right)+\left(\begin{array}{c}
2 D+2 \\
Q+1
\end{array}\right)\right. \\
& \left.\left.-(N-D-2)\left(\begin{array}{c}
D+1 \\
Q
\end{array}\right)-\left(\begin{array}{c}
D+2 \\
Q+1
\end{array}\right)\right]\right\} \\
= & \frac{1}{\left(\begin{array}{c}
N \\
Q
\end{array}\right)}\left\{\frac{D Q-D-2}{Q+1}\left(\begin{array}{c}
2 D+1 \\
Q
\end{array}\right)+\left(\begin{array}{c}
D+2 \\
Q+1
\end{array}\right)\right\} .
\end{aligned}
$$

We proceed now to examine the second subcase. The expected head movement is

$$
\begin{aligned}
& \sum_{K=N-2 D-1}^{N-D-2} P(N, Q, K) \sum_{I=1}^{2 D-N+2+K} \frac{\left(\begin{array}{c}
2 D-N+2+K \\
I
\end{array}\right)\left(\begin{array}{c}
2 N-2 K-2 D-3 \\
Q-I-1
\end{array}\right)}{\left(\begin{array}{c}
N-K-1 \\
Q-1
\end{array}\right)} \\
& \sum_{M=0}^{2 D-N+1+K}(2 D-N+2+K-M) P(2 D-N+2+K, I, M)=\mathrm{C} .
\end{aligned}
$$

The second summation gives the probability that $I$ requests are distributed over $2 D-N+2+K$ cylinders and the remaining $Q-I-1$ requests are distributed 
over $2 N-2 K-2 D-3$ cylinders.

$$
\begin{aligned}
& \mathrm{C}=\sum_{K=N-2 D-1}^{N-D-2} \frac{\left(\begin{array}{c}
N-1-K \\
Q-1
\end{array}\right)}{\left(\begin{array}{l}
N \\
Q
\end{array}\right)} \sum_{I=1}^{2 D-N+2+K} \frac{\left(\begin{array}{c}
2 D-N+2+K \\
I
\end{array}\right)\left(\begin{array}{c}
2 N-2 K-2 D-3 \\
Q-I-1
\end{array}\right)}{\left(\begin{array}{c}
N-K-1 \\
Q-1
\end{array}\right)} \\
& \times \sum_{M=0}^{2 D-N+1+K}(2 D-N+2+K-M) \frac{\left(\begin{array}{c}
2 D-N+1+K-M \\
I-1
\end{array}\right)}{\left(\begin{array}{c}
2 D-N+2+K \\
I
\end{array}\right)} \\
& =\frac{1}{\left(\begin{array}{l}
N \\
Q
\end{array}\right)} \sum_{K=N-2 D-1}^{N-D-2} \sum_{I=1}^{2 D-N+2+K}\left(\begin{array}{c}
2 N-2 K-2 D-3 \\
Q-1-I
\end{array}\right) \\
& \times \sum_{M=0}^{2 D-N+1+K}(2 D-N+2+K-M)\left(\begin{array}{c}
2 D-N+1+K-M \\
I-1
\end{array}\right) \\
& =\frac{1}{\left(\begin{array}{l}
N \\
Q
\end{array}\right)}\left\{\sum_{K=N-2 D-1}^{N-D-2} \sum_{I=1}^{2 D-N+2+K}\left(\begin{array}{c}
2 N-2 K-2 D-3 \\
Q-1-I
\end{array}\right)\right. \\
& {\left[(2 D-N+2+K) \sum_{M=0}^{2 D-N+1+K}\left(\begin{array}{c}
2 D-N+1+K-M \\
I-1
\end{array}\right)\right.} \\
& \left.-\sum_{M=0}^{2 D-N+1+K} M\left(\begin{array}{c}
2 D-N+1+K-M \\
I-1
\end{array}\right)\right\} .
\end{aligned}
$$

By substitution of (4) and (5) we derive that

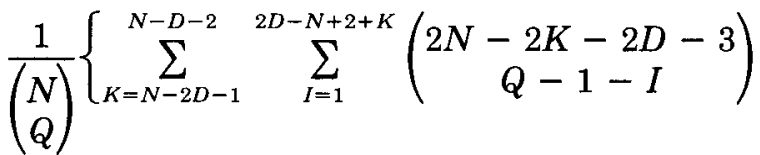

$$
\begin{aligned}
& \left.\left[(2 D-N+2+K)\left(\begin{array}{c}
2 D-N+K+2 \\
I
\end{array}\right)-\left(\begin{array}{c}
2 D-N+K-2 \\
I+1
\end{array}\right)\right]\right\} \\
& =\frac{1}{\left(\begin{array}{l}
N \\
Q
\end{array}\right)}\left\{\sum_{K=N-2 D-1}^{N-D-2}\{(2 D-N+2+K)\right. \\
& \times\left[\sum_{I=0}^{2 D-N+2+K}\left(\begin{array}{c}
2 N-2 K-2 D-3 \\
Q-1-I
\end{array}\right)\left(\begin{array}{c}
2 D-N+K+2 \\
I
\end{array}\right)\right. \\
& -\left(\begin{array}{c}
2 N-2 K-2 D-3 \\
Q-1
\end{array}\right) \\
& -\left[\sum_{I=-1}^{2 D-N+2+K}\left(\begin{array}{c}
2 N-2 K-2 D-3 \\
Q-1-I
\end{array}\right)\left(\begin{array}{c}
2 D-N+K+2 \\
I
\end{array}\right)\right. \\
& \left.\left.\left.-\sum_{I=-1}^{0}\left(\begin{array}{c}
2 N-2 K-2 D-3 \\
Q-1-I
\end{array}\right)\left(\begin{array}{c}
2 D-N+K+2 \\
I
\end{array}\right)\right]\right\}\right\} .
\end{aligned}
$$


By substitution of (6) we have that

$$
\begin{aligned}
& \frac{1}{\left(\begin{array}{l}
N \\
Q
\end{array}\right)}\left\{\sum _ { K = N - 2 D - I } ^ { N - D - 2 } \left\{(2 D-N+2+K)\left[\left(\begin{array}{c}
N-1-K \\
Q-1
\end{array}\right)-\left(\begin{array}{c}
2 N-2 K-2 D-3 \\
Q-1
\end{array}\right)\right]\right.\right. \\
& -\left[\left(\begin{array}{c}
N-1-K \\
Q
\end{array}\right)-\left(\begin{array}{c}
2 N-2 K-3-2 D \\
Q
\end{array}\right)\right. \\
& \left.\left.\left.-(2 D-N+K+2)\left(\begin{array}{c}
2 N-2 K-3-2 D \\
Q-1
\end{array}\right)\right]\right\}\right\} \\
& =\frac{1}{\left(\begin{array}{l}
N \\
Q
\end{array}\right)}\left\{\sum_{K=N-2 D-1}^{N-D-2}\left(2 D+3-\frac{(N-K)(Q+1)}{Q}\right)\left(\begin{array}{c}
N-1-K \\
Q-1
\end{array}\right)\right. \\
& \left.+\left(\begin{array}{c}
2 N-2 K-2 D-3 \\
Q
\end{array}\right)\right\} \\
& =\frac{1}{\left(\begin{array}{l}
N \\
Q
\end{array}\right)}\left\{\left(2 D+3-\frac{N(Q+1)}{Q}\right) \sum_{K=N-2 D-1}^{N-D-2}\left(\begin{array}{c}
N-1-K \\
Q-1
\end{array}\right)\right. \\
& +\frac{Q+1}{Q} \sum_{K=N-2 D-1}^{N-D-2} K\left(\begin{array}{c}
N-1-K \\
Q-1
\end{array}\right) \\
& \left.+\sum_{K=N-2 D-1}^{N-D-2}\left(\begin{array}{c}
2 N-2 K-2 D-3 \\
Q
\end{array}\right)\right\} \\
& =\frac{1}{\left(\begin{array}{c}
N \\
Q
\end{array}\right)}\left\{\left(2 D+3-\frac{N(Q+1)}{Q}\right)\left[\left(\begin{array}{c}
2 D+1 \\
Q
\end{array}\right)-\left(\begin{array}{c}
D+1 \\
Q
\end{array}\right)\right]\right. \\
& +\frac{Q+1}{Q}\left[(N-2 D-2)\left(\begin{array}{c}
2 D+1 \\
Q
\end{array}\right)+\left(\begin{array}{c}
2 D+2 \\
Q+1
\end{array}\right)\right. \\
& \left.-(N-D-2)\left(\begin{array}{c}
D+1 \\
Q
\end{array}\right)-\left(\begin{array}{c}
D+2 \\
Q+1
\end{array}\right)\right] \\
& \left.+\sum_{K=N-2 D-1}^{N-D-2}\left(\begin{array}{c}
2 N-2 K-2 D-3 \\
Q
\end{array}\right)\right\} \\
& =\frac{1}{\left(\begin{array}{l}
N \\
Q
\end{array}\right)}\left\{\sum_{K-N-2 D-2}^{N-D-2}\left(\begin{array}{c}
2 N-2 K-2 D-3 \\
Q
\end{array}\right)-(D+1)\left(\begin{array}{c}
D+1 \\
Q
\end{array}\right)\right\} .
\end{aligned}
$$

If $N-2 D-2 \geq 0$, then by summing formulas (7), (9), (12), and (14), finally we derive that the expected arm movement is

$$
\frac{1}{\left(\begin{array}{l}
N \\
Q
\end{array}\right)}\left\{\left(\frac{(N+1) Q}{Q+1}-D-2\right)\left(\begin{array}{l}
N \\
Q
\end{array}\right)+\sum_{K=N-2 D-2}^{N-D-2}\left(\begin{array}{c}
2 N-2 K-2 D-3 \\
Q
\end{array}\right)\right\} .
$$


If $N-2 D-2<0$ but $N-D-2>0$, then by modifying (10) and (13) and summing ( 7 ) we derive that the expected head movement is

$$
\begin{aligned}
E=\frac{1}{\left(\begin{array}{l}
N \\
Q
\end{array}\right)}\{ & \frac{(D+1) Q-1}{Q+1}\left(\begin{array}{c}
D+1 \\
Q
\end{array}\right)+\sum_{K=0}^{N-D-2}(N-D-2-K)\left(\begin{array}{c}
N-1-K \\
Q-1
\end{array}\right) \\
& +\left(2 D+3-\frac{N(Q+1)}{Q}\right) \sum_{K=0}^{N-D-2}\left(\begin{array}{c}
N-1-K \\
Q-1
\end{array}\right) \\
& \left.+\frac{Q+1}{Q} \sum_{K=1}^{N-D-2} K\left(\begin{array}{c}
N-1-K \\
Q-1
\end{array}\right)+\sum_{K=0}^{N-D-2}\left(\begin{array}{c}
2 N-2 K-2 D-3 \\
Q
\end{array}\right)\right\} .
\end{aligned}
$$

We simplify the second term. Without loss of generality let $Q<D+3$. Then:

$$
\begin{aligned}
& \sum_{K=0}^{N-D-2}(N-D-2-K)\left(\begin{array}{c}
N-1-K \\
Q-1
\end{array}\right) \\
& =(N-D-2)\left[\sum_{K=0}^{N-Q}\left(\begin{array}{c}
N-1-K \\
Q-1
\end{array}\right)-\sum_{K=N-D-1}^{N-Q}\left(\begin{array}{c}
N-1-K \\
Q-1
\end{array}\right)\right] \\
& -\left[\sum_{K=1}^{N-Q} K\left(\begin{array}{c}
N-1-K \\
Q-1
\end{array}\right)-\sum_{K=N-D-1}^{N-Q} K\left(\begin{array}{c}
N-1-K \\
Q-1
\end{array}\right)\right] \\
& =(N-D-2)\left[\left(\begin{array}{l}
N \\
Q
\end{array}\right)-\left(\begin{array}{c}
D+1 \\
Q
\end{array}\right)\right] \\
& -\left[\left(\begin{array}{c}
N \\
Q+1
\end{array}\right)-(N-D-2)\left(\begin{array}{c}
D+1 \\
Q
\end{array}\right)-\left(\begin{array}{c}
D+1 \\
Q+1
\end{array}\right)\right] \\
& =\left(\frac{(N+1) Q}{Q+1}-D-2\right)\left(\begin{array}{l}
N \\
Q
\end{array}\right)+\left(\begin{array}{l}
D+2 \\
Q+1
\end{array}\right) \text {. }
\end{aligned}
$$

Again, without loss of generality let $Q \leq D+2$. Then the third and fourth terms are simplified in the following way:

$$
\begin{aligned}
& \left(2 D+3-\frac{N(Q+1)}{Q}\right)\left[\sum_{K=0}^{N-Q}\left(\begin{array}{c}
N-1-K \\
Q-1
\end{array}\right)-\sum_{K=N-D-1}^{N-Q}\left(\begin{array}{c}
N-1-K \\
Q-1
\end{array}\right)\right] \\
& +\frac{Q+1}{Q}\left[\sum_{K=1}^{N-Q} K\left(\begin{array}{c}
N-1-K \\
Q-1
\end{array}\right)-\sum_{K=N-D-1}^{N-0} K\left(\begin{array}{c}
N-1-K \\
Q-1
\end{array}\right)\right] \\
& =\left(2 D+3-\frac{N(Q+1)}{Q}\right)\left[\left(\begin{array}{c}
N \\
Q
\end{array}\right)-\left(\begin{array}{c}
D+1 \\
Q
\end{array}\right)\right] \\
& +\frac{Q+1}{Q}\left[\left(\begin{array}{c}
N \\
Q+1
\end{array}\right)-(N-D-2)\left(\begin{array}{c}
D+1 \\
Q
\end{array}\right)-\left(\begin{array}{c}
D+2 \\
Q+1
\end{array}\right)\right] \\
& =(2 D+2-N)\left(\begin{array}{l}
N \\
Q
\end{array}\right)-(D+1)\left(\begin{array}{c}
D+1 \\
Q
\end{array}\right) \text {. }
\end{aligned}
$$

ACM Transactions on Database Systems, Vol. 14, No. 3, September 1989. 
The last term cannot be simplified. Therefore finally:

$$
\mathrm{E}=\frac{1}{\left(\begin{array}{l}
N \\
Q
\end{array}\right)}\left[\left(\frac{(N+1) Q}{Q+1}+D-N\right)\left(\begin{array}{l}
N \\
Q
\end{array}\right)+\sum_{K=0}^{N-D-2}\left(\begin{array}{c}
2 N-2 K-2 D-3 \\
Q
\end{array}\right)\right\} .
$$

Therefore, the expected distance traveled by the disk heads is given by formula (17) which unifies (15) and (16):

$$
\begin{gathered}
D T=\frac{1}{\left(\begin{array}{c}
N \\
Q
\end{array}\right)}\left\{\left(\frac{(N+1) Q}{Q+1}-D-2+f(2 D+2-N)\right)\left(\begin{array}{l}
N \\
Q
\end{array}\right)\right. \\
\left.+\sum_{K=f(N-2 D-2)}^{N-D-2}\left(\begin{array}{c}
2 N-2 K-2 D-3 \\
Q
\end{array}\right)\right\}
\end{gathered}
$$

where the function $f(\cdot)$ is defined as

$$
f(a)=\left\{\begin{array}{lll}
a & \text { if } & a>0 \\
0 & \text { if } & a \leq 0
\end{array}\right.
$$

\section{OPTIMUM HEAD SEPARATION AND COMPARISON WITH ONE-HEADED SYSTEMS}

Figures 2 and 3 consider a file occupying 75 and 100 cylinders, respectively, and have been produced by applying formula (17) for a range of values of the parameters $Q$ and $D$. From these figures and many other similar figures we produced, we observed that the optimum head separation is $N / 2-1(\lceil N / 2-11$ and $L N / 2-1 \mathrm{~J}$ ) cylinders, if $N$ is even (odd). Moreover, we observed that all the curves have an axis of symmetry. The following theorems prove that the two observations are true.

THEOREM 1. If $N$ is even, then the head separation distances, which differ the same quantity from $N / 2-1$, result in the same expected distance traveled as derived by relation (17).

Proof. Without loss of generality, we assume that $N$ is even, but a similar argument holds for the case that $N$ is odd.

We are going to prove that the expected distances traveled by the moving arm, when the head separation distances are D1 $=N / 2-1-a$ and D2 $=$ $N / 2-1+a$, are equal. Evidently, the quantity $a$ is an integer value and the following relations hold: $\mathrm{D} 2-\mathrm{D} 1=2 a$ and $0<a<N / 2-1$. From relation (17) with simple algebra we derive that

$$
\begin{aligned}
& D T(D 1)=\frac{1}{\left(\begin{array}{l}
N \\
Q
\end{array}\right)}\left\{\left(\frac{(N+1) Q}{Q+1}-\frac{N}{2}+a-1\right)\left(\begin{array}{l}
N \\
Q
\end{array}\right)+\sum_{K=2 a}^{N / 2-1+a}\left(\begin{array}{c}
N-2 K-1+2 a \\
Q
\end{array}\right)\right\} . \\
& D T(D 2)=\frac{1}{\left(\begin{array}{l}
N \\
Q
\end{array}\right)}\left\{\left(\frac{(N+1) Q}{Q+1}-\frac{N}{2}+a-1\right)\left(\begin{array}{l}
N \\
Q
\end{array}\right)+\sum_{K=0}^{N / 2-1-a}\left(\begin{array}{c}
N-2 K-1-2 a \\
Q
\end{array}\right)\right\} .
\end{aligned}
$$




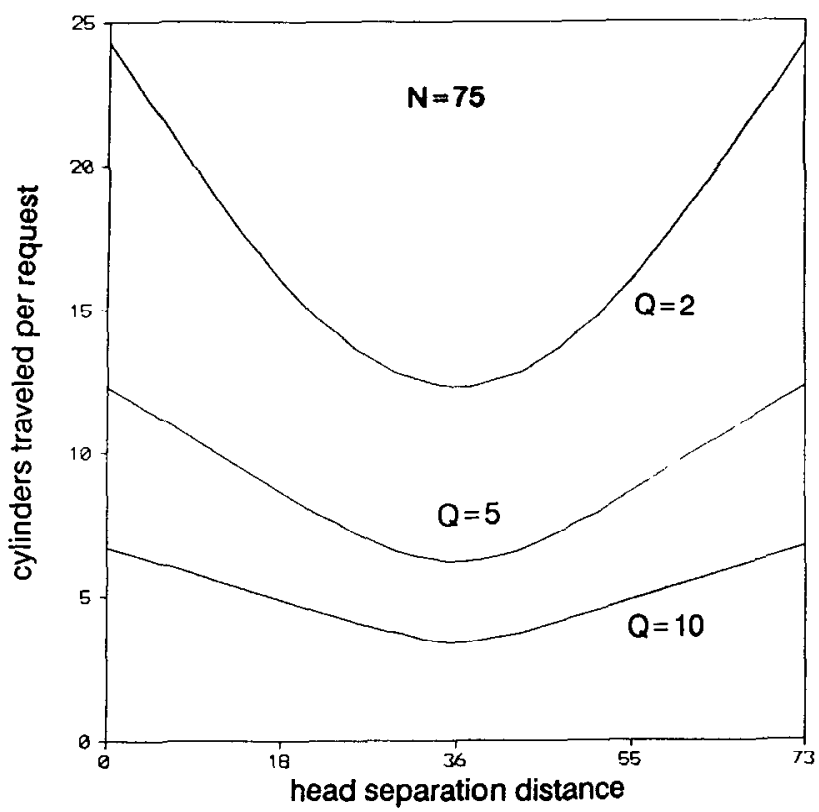

Fig. 2. Expected distance traveled per request as a function of the head separation distance. The file is stored in 75 consecutive cylinders, and the magnitude of the query is 2,5 , and 10 records.

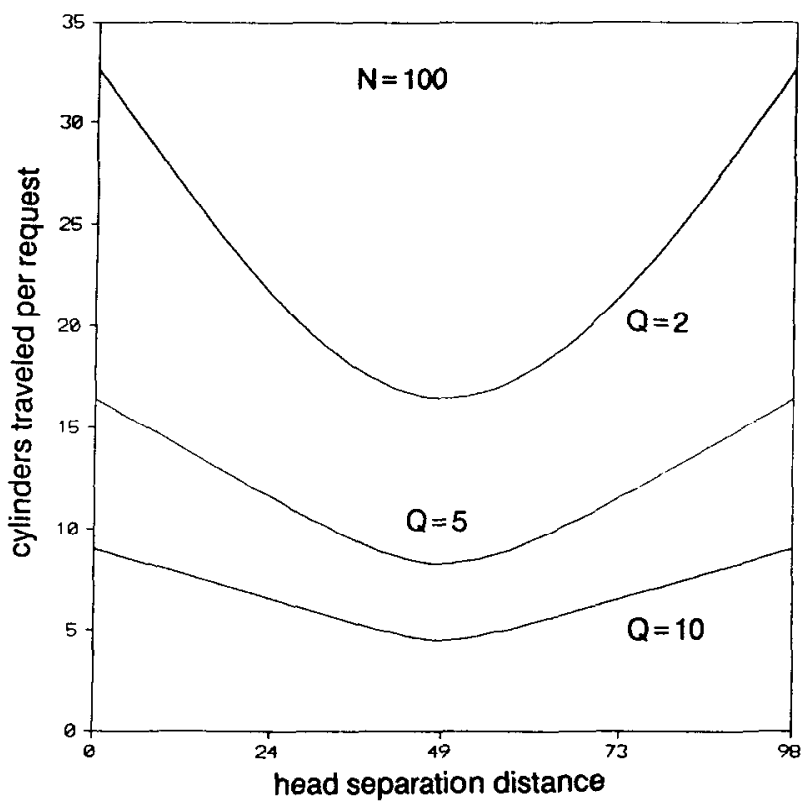

Fig. 3. Expected distance traveled per request as a function of the head separation distance. The file is stored in 100 consecutive cylinders, and the magnitude of the query is 2,5 , and 10 records.

ACM Transactions on Database Systems, Vol. 14, No. 3, September 1989. 
Obviously, $D T(D 1)$ equals $D T(D 2)$ if and only if

$$
\sum_{K=2 a}^{N / 2-1+a}\left(\begin{array}{c}
N-2 K-1+2 a \\
Q
\end{array}\right)=\sum_{K=0}^{N / 2-1-a}\left(\begin{array}{c}
N-2 K-1-2 a \\
Q
\end{array}\right) .
$$

It can easily be shown by simply replacing the values of $K$ and expanding the sums that this relation is true.

If $N$ is odd, then in a similar manner it can be shown that the separation distances, which result in the same expected distance traveled by the moving arm, are given by relations $D 1=\lceil N / 2-1\rceil+a$ and $D 2=\lfloor N / 2-1\rfloor-a$.

THEOREM 2. The optimum head separation is $N / 2-1(\lceil N / 2-11$ and $\mathrm{L} N / 2-1 \mathrm{~J}$ ) cylinders if $N$ is even (odd).

Proof. Again, without loss of generality, we assume that $N$ is even. It is sufficient to prove that $D T(N / 2-1)<D T(N / 2-1+a)$.

$$
D T(N / 2-1)=\frac{1}{\left(\begin{array}{l}
N \\
Q
\end{array}\right)}\left(\frac{(N+1) Q}{Q+1}-\frac{N}{2}-1\right)\left(\begin{array}{c}
N \\
Q
\end{array}\right)+\sum_{K=0}^{N / 2-1}\left(\begin{array}{c}
N-1-2 K \\
Q
\end{array}\right) .
$$

Therefore:

$$
\begin{aligned}
& D T(N / 2-1+a)-D T(N / 2-1) \\
& =\frac{1}{\left(\begin{array}{l}
N \\
Q
\end{array}\right)}\left\{a\left(\begin{array}{l}
N \\
Q
\end{array}\right)+\sum_{K=2 a}^{N / 2-1-a}\left(\begin{array}{c}
N-2 K-1-2 a \\
Q
\end{array}\right)-\sum_{K=0}^{N / 2-1}\left(\begin{array}{c}
N-1-2 K \\
Q
\end{array}\right)\right\} .
\end{aligned}
$$

By expanding the sums and eliminating the equal terms we derive

$$
\frac{1}{\left(\begin{array}{l}
N \\
Q
\end{array}\right)}\left\{a\left(\begin{array}{l}
N \\
Q
\end{array}\right)-\sum_{K=N / 2-a}^{N / 2-1}\left(\begin{array}{c}
N-2 K-1-2 a \\
Q
\end{array}\right)\right\} .
$$

The last sum consists of $a$ terms. Each of these terms is smaller than the combination $\left(\begin{array}{c}N \\ Q\end{array}\right)$. Therefore, the last quantity is always positive.

Relation (17) is not a continuous function. The authors used Stirling's approximate formula to replace combinations and assumed that the function is continuous. However, they did not succeed in finding an easy way to evaluate a closed form formula for the optimum $D$ as a function of $N$.

As we have seen, the expected head movement to answer a user query involving $Q$ cylinders in a single-headed system with $N$ cylinders is given by (2). The equivalent case, in which the two heads are positioned on the top of the same cylinder, may be expressed by dropping, for the moment, the assumption that $O \leq D \leq N-2$ and substituting $D$ by -1 in relation (17). In this case the last relation degenerates to relation (2). Therefore, this observation unifies and validates both approaches. Figure 4 shows the reduction in the number of cylinders traveled because there are two heads per moving arm in a system with 100 cylinders. The $X$-axis is the number of cylinder hits, and the $Y$-axis is the number of cylinders traveled. The lower curve is drawn by applying formula (17) 


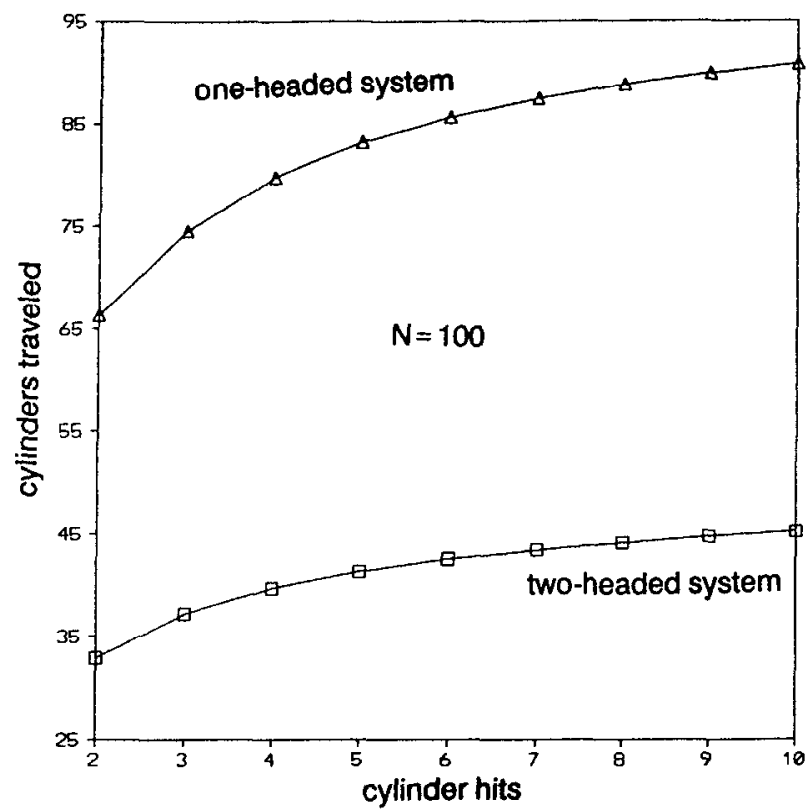

Fig. 4. Comparison between a onc-headed and a two-headed system, with the expected distance traveled as a function of the magnitude of the query. The file is stored in 100 consecutive cylinders.

for $D=49$ (optimal head separation distance). The figure shows that the reduction is more than 50 percent. If $D \neq 49$, then the resulting curve lies in between the two drawn curves.

When evaluating scheduling policies, the most important metric is the seek time. However the quantities $S \min$ and $S \max$ of formula (1) are hardware dependent. By giving values to these quantities, additional figures of seek time versus cylinder hits would be drawn but no more insight into the system could be achieved. Nevertheless, even if seek time is the cost metric, it is clear that formula (21) is still valid because the product $Q * S \min$ in expression (1) is not a function of the disk separation distance $D$. Therefore, the derivative of $T$ is again equal to (20). Because of the difficulty of aligning multiple heads to the disk tracks, it is anticipated that the reduction in that case would be considerably less than 50 percent.

\section{CONCLUSIONS}

This study analyzed the performance of a two-headed disk system when the SCAN policy is used to answer user queries from the file. In particular, one exact expression of the expected head movement was derived. This expression may augment the list of formulas referring to similar estimates but for one-headed systems $[1,2,15,18,24]$. The expression was subsequently used to prove that the optimal head separation is $N / 2-1(\lceil N / 2-11$ and $L N / 2-1\rfloor)$ if the file size in cylinders, $N$, is even (odd). This last result extends the work in [3] and 
[20] which also consider the problem of determining the optimal head separation for other environments. This conclusion is valid under the assumption that either of the two heads may move outside the recording area. In [20] almost the same conclusion was met after the assumption that the two heads are not allowed to pass over the extreme cylinders. In this way, our analytical point of view extends the results of [20].

Comparison with a single-headed system operating under the same scheduling algorithm shows that the reduction in disk head movement is more than 50 percent when the disk heads are optimally spaced. This result may be of value to database practitioners because (a) two-headed disk systems have already appeared on the market, and (b) the SCAN policy is useful for a number of file processing usages.

The study may be extended in a number of directions. We mention some of them. First, simulation techniques may be used to evaluate the performance of a two-headed disk system in terms of total seek time. The simulation model may also consider other important issues of the problem (for example, the overhead involved to initiate one seek) with the objective of answering questions such as: "If I buy 5 one-headed disks for the price of 3 two-headed disks, do I get a lower average seek time from the one-headed disks or the two-headed disks?" Second, the optimal query satisfaction sequence may be investigated in the case that more than one (batch of) queries are to be served in succession by the SCAN policy $[2,17,19]$. Third, new file organization schemes may be derived that take advantage of the two disk heads. Fourth, modification of the studies in [10], [21], and [23] could determine the optimal reorganization points for files residing in two-headed disk systems. Last, study disk systems with two independent moving arms [13]. In particular, comparison of their performance to that of the twoheaded disk systems with heads at a fixed distance may lead to a proposition of new efficient scheduling algorithms.

\section{ACKNOWLEDGMENT}

We would like to thank the referees for their helpful comments and suggestions which improved the quality of the presentation.

\section{REFERENCES}

1. ANDERSON, H. D., AND BerRA, P. B. Minimum cost selection of secondary indexes for formatted files. ACM Trans. Database Syst. 2, 1 (Mar. 1977), 68-90.

2. Burton, F. W., AND Kollias, J. G. Optimizing disk head movement in secondary key retrieval. Comput. J. 22, 3 (Aug. 1979), 206-208.

3. Calderbank, A. R., Coffman, E. G.,And Flatto, L. Optimum head separation in a disk system with two read/write heads. J. ACM 31, 4 (Oct. 1984), 826-838.

4. Calderbank, A. R., Coffman, E. G., and Flatto, L. Optimal directory placement on disk storage devices. J. ACM 35, 2 (Apr. 1988), 433-446.

5. Cardenas, A. F. Analysis and performance of inverted database structures. Commun. ACM 18, 5 (May 1975), 253-263.

6. Cheung, T. Y. Estimating block accesses and number of records in file management. Commun. ACM 25, 7 (July 1982), 484-486.

7. Christodoulakis, S. Estimating block transfers and join sizes. In Proceedings of the ACM SIGMOD Conference (San Jose, Calif., May 23-26, 1983). ACM, New York, 1983, pp. 40-54. 
8. Christodoulakis, S. Implications of certain assumptions in database performance evaluation. ACM Trans. Database Syst. 9, 2 (June 1984), 163-186.

9. Christodoulakis, S. Analysis of retrieval performance for records and objects using optical disk technology. ACM Trans. Database Syst. 12, 2 (June 1987), 137-169.

10. Date, C. J. An Introduction to Database Systems, 3rd ed. Addison-Wesley, Reading, Mass., 1981.

11. Feller, W. An Introduction to Probability Theory and its Applications, 3rd ed. Wiley, New York, 1968.

12. Hatzopoulos, M., and Kollias, J. G. The determination of the optimum database reorganization points. Comput. J. 25, 1 (Feb. 1982), 126-129.

13. Hofri, M. Should the two-headed disk be greedy? Yes, it should. Inf. Proces. Lett. 16 (Feb. 1983), 83-85.

14. KNUth, D. The Art of Computer Programming. Vol. 3, Sorting and Searching. Addison-Wesley, Reading, Mass., 1973.

15. Kollias, J. G. An estimate of seek time for batched searching of random or indexed sequential structured files. Comput. J. 21, 2 (May 1978), 132-133.

16. Kollias, J. G., Manolopoulos, Y., and Papadimitriou, C. The optimal execution order of queries in linear storage. Submitted for publication.

17. Manolopoulos, Y., AND Kollias, J. G. Placement of records in two-headed disk systems. Submitted for publication.

18. Manolopoulos, Y., ANd Kollias, J. G. Estimating disk head movement in batched searching. BIT 28 (1988), 27-36.

19. MANOlopoulos, Y. Probability distributions for seek time evaluation. Inf. Sci. In press.

20. PAge, I. P., AND Wood, R. T. Empirical analysis of a moving headed disk model with two heads separated by a fixed number of tracks. Comput. J. 24, 4 (Nov. 1981), 339-342.

21. Shneiderman, B. Optimum database reorganization points. Commun. ACM 16, 6 (June 1973), 362-365.

22. Shneiderman, B., and Goodman, V. Batched searching of sequential and tree structured files. ACM Trans. Database Syst. 1, 3 (Sept. 1976), 268-275.

23. Tues, W. G. Optimum reorganization points for linearly growing files. ACM Trans. Database Syst. 3, 1 (Mar. 1978), 32-40.

24. Waters, S. J. Estimating magnetic disk seeks. Comput. J. 18, 1 (Feb. 1975), $12-19$.

25. Wiederhold, G. File Organizations for Database Design. McGraw-Hill, New York, 1987.

26. Wong, C. K. Minimizing expected head movement in one-dimensional and two-dimensional mass storage systems. ACM Comput. Surv. 12, 2 (June 1980), 167-178.

27. YAO, S. B. An attribute based model for database cost analysis. ACM Trans. Database Syst. 2, 1 (Mar. 1977), 45-67.

28. YAO, S. B. Approximating block accesses in database organizations. Commun. ACM 20, 4 (Apr. 1977), 260-261.

Received March 1986; revised September 1987; accepted November 1988 